\title{
STUDY OF THE POSSIBLE RELATIONSHIPS BETWEEN TRAMWAY FRONT-END GEOMETRY AND PEDESTRIAN INJURY RISK
}

\author{
Marie-Christine Chevalier, Denis Brizard, Philippe Beillas \\ Univ Lyon, Université Claude Bernard Lyon 1, IFSTTAR, LBMC UMR_T9406, F69622, Lyon, France
}

\begin{abstract}
(200-400 words)
Objectives: The aim of this paper is to report on the possible relationships between tramway front end geometry and pedestrian injury risk over a wide range of possible tramway shapes.

Methods: To study the effect of tramway front-end shape on pedestrian injury metrics, accidents were simulated using a custom parametrized model of tramway front-end and pedestrian models available with the MADYMO multibody solver. The approach was automated, allowing the systematic exploration of tramway shapes in conjunction with four pedestrian sizes (e.g. $50^{\text {th }}$ percentile male or M50).
\end{abstract}

Results: A total of 8,840 simulations were run, showing that the injury risk is more important for the head than for the other body regions (thorax and lower extremities). The head of the M50 impacted the windshield of the tramway in most of the configurations. Two antagonist mechanisms affecting impact velocity of the head and corresponding HIC values were observed. The first is a trunk rotation resulting from an engagement of the lower body that can contribute to an increase of the head velocity in the direction of the tram. The second is the loading of the shoulder that can accelerate the upper trunk and head away from the windshield, resulting in lower impact velocities. Groups of design were defined based on two main parameters (windshield height and offset), some of which seem more beneficial than others for tramway design. The pedestrian size and tramway velocity $(30 \mathrm{~km} / \mathrm{h}$ vs. $20 \mathrm{~km} / \mathrm{h})$ also affected the results.

Conclusions: When considering only the frontend shape, the best strategy to limit the risk of head injury due to the contact with the stiff windshield seems to be to promote the mechanism involving shoulder loading. As body regions engaged vary with the pedestrian size, none of the groups of designs performed equally well for all pedestrian sizes. The best compromise seems achieved with a combination of a large windscreen offset and a high windscreen. Conversely, particularly unfavorable configurations are observed for low windshield heights, especially with a large offset. Beyond the frontend shape, considering the stiffness of the current windshields and the high injury risks predicted for $30 \mathrm{~km} / \mathrm{h}$, the stiffness of the windshield should be considered in the future for further gains in pedestrian safety.

\section{Keywords for indexing}

impact biomechanics; injury mechanisms; multibody modelling; vulnerable road users; tramway design

\section{INTRODUCTION}

Accidents between tramways and pedestrians are a well-known problem (Demant et al. 2010) which could gain further significance as the tramway networks are being extended in many cities. For example, in France, between 2006 and 2015, the number of kilometers traveled by the tramways increased from 30 to 72 million km (as reported by the French Technical Agency for Ropeways and Guided Transport Systems (STRMTG) (de Labonnefon and Passelaigue 2016)). Concurrently, the number of accidents involving road users increased from 971 events to 1472. While accidents with 
pedestrians represented only $15 \%$ of the events in 2015 , pedestrians were about half of the road users injured by tramways. These cases led to four fatalities in 2015, and 17 in the period [2012-2015]. In Gothenburg (Sweden) during the period 1988-2002, 26 (48\%) of 54 pedestrians involved in an accident with a tramway were killed (Hedelin, et al. 1996). Pedestrian safety has been extensively studied in the context of accidents with cars (Kerrigan et al. 2007; Subit et al. 2008; Kerrigan et al. 2009; Fredriksson 2011; Watanabe et al. 2012) and to a lesser extent with trucks (Feist et al. 2009; Beillas et al. 2011; Matsui et al. 2017). Studies have shown that the shape and stiffness of the front-end of the vehicle, as well as the impact velocity, have a great influence on both the kinematics and injuries of the pedestrian. Consequently, regulations and consumer tests have been designed to assess the car front-end performance (Regulation (EC) No 78 2009; EuroNCAP 2016) but there are no equivalents for the tramways.

As a wide variety of tramway shapes (e.g. in terms of angles, curvature, and windshield location) seem possible based on field observations, pedestrian protection may be used as one of the criteria to help design future front shapes. This could be especially relevant for tramways as they can be subjected to regulations leading to very stiff windshields. For example, in France, NF-F15818 1996 requires tramway windshields not to fail when impacted by a 22LR bullet shot at over $290 \mathrm{~m} / \mathrm{s}$ and by a $1 \mathrm{~kg}$ mass launched at $140 \mathrm{~km} / \mathrm{h}$ (Type 1 glass for an assumed maximum tramway velocity of $70 \mathrm{~km} / \mathrm{h}$ ). A few studies have proposed methods to design more "pedestrian friendly" tramway front-ends. In (Grzebieta and Rechnitzer 2000), two Australian tramway front-ends with more or less protruding steel bumper bars were modelled with MADYMO multibody solver (TASS 2013) and a pedestrian impact was simulated using a Hybrid III dummy in standing position. The impact velocity was $35 \mathrm{~km} / \mathrm{h}$ and injuries due to the contact with the floor were not considered. The front-end with the most protruding bumper had the highest risk of injury: impacted by the bumper, the pedestrian rotated around the thigh, causing the head to strike a stiff area of the tramway front-end just below the windshield (Head Injury Criteria or HIC>1000). Padding added between the bumper and the windshield helped to reduce the HIC. For the other front-end, which was more flat, the pedestrian was pushed forward without such rotation, leading to a lower HIC value. In (Hyncik et al. 2008), a 50 ${ }^{\text {th }}$ percentile male model and a 6-year old child model were struck by a Czech front tramway model at $15 \mathrm{~km} / \mathrm{h}$. The simulation results suggested that the bumper should be placed at a lower position for the adult pedestrian than for the child. More recently, investigations performed in Switzerland were used to derive recommendations for tramway front-end design in collaboration with Swiss public transport services (Weber et al. 2015). As the windshield of trams needs to have a high stiffness, the study recommended to avoid the head impact against this element or at least to reduce the head impact velocity. It suggested that a velocity reduction could be achieved by combining a forward position of the front trim panel (fairing) relative to the windshield by about $8-10 \mathrm{~cm}$ and a high position of $1 \mathrm{~m} 30$ for the lower edge of the windshield. Overall, these studies show that the shape of the tramway front-end affects the risk of pedestrian head injuries. However their limitations included the fact that they were typically variations around a baseline front-end design: they did not attempt to assess the effects of front-end shape through a more general exploration of the design space, i.e. accounting for the front-end variability (face inclination, windshield angle, etc.), as well as the effect of the pedestrian size.

The aim of this paper is to report (1) on the possible relationships between tramway front-end geometry and pedestrian injury risk over a wide range of possible tramway shapes, and (2) on the methodology developed to study them. This work was performed at IFSTTAR at the request of STRMTG in order to support the activities of a working group aiming to formulate tramway design requirements that would better account for pedestrian passive safety. The working group is composed of the STRMTG as well tramway manufacturers and operators (see appendix 1). 


\section{TOOLS AND METHODS}

\section{Scope and overview}

Based on the requirements to describe a variety of possible tramway shapes and on previous studies in the literature (Soni et al. 2013; Martinez et al. 2007; Van Rooij et al. 2003), a simulation approach using the MADYMO software was selected for its computational efficiency. A parametrized model of tramway front-end was developed and used along with the pedestrian models provided with MADYMO. The tramway velocity was initially defined at $20 \mathrm{~km} / \mathrm{h}$. Parameters considered in the parametric study included angles and dimensions for the tramway front-end, and pedestrian sizes. The effect of the impact velocity was considered for the medium adult. The effects of windshield or fairing stiffness were outside the scope of the study. Only injuries resulting from the primary impact were considered. For the analysis, as the goals of the application were to help avoid the most dangerous configurations and to define simple geometric rules without completely constraining the design, groups were defined based on the parameters having the most effect on the response. More details are provided below.

\section{Pedestrian Models}

Four body sizes of the pedestrian MADYMO ellipsoid model (TNO 2013) were used. Each model consists of 52 rigid bodies organized in seven branches representing the head-thorax, arms, legs and heel-mid foot regions. Articulations are modelled by kinematic joints and sensors are implemented for the assessment of injury criteria. (Van Hoof et al. 2003) evaluated the model performance through blunt impact tests and pedestrian-car tests.

The mid-size male model (50th percentile male stature, later denoted M50) was used as the baseline with all combinations of parameters. In order to assess the influence of the pedestrian size, simulations were also performed with the small female model (5th percentile female, F05), the large male model (95th percentile male, M95) and the child model (6 year-old child, C06).

\section{Simplified Model of the Tramway Front-end and Baseline Simulation Setup}

Tramway front-end designs can vary widely in the field in terms of face inclination (from vertical to about $45^{\circ}$ ), local surface shape (plane, angular or smooth with continuously varying curvature), height of the windshield, etc.

The windshield typically covers most of the upper part of the front-end while the fairing covers the lower. In France, the windshield has to follow railway strength regulations (NF-F15818 1996), making it a very rigid component. In modern tramways, the fairing is typically a plastic or composite shell covering stiffer structures such as shock absorbers and coupling devices.

In order to limit the number of parameters, the configuration was idealized as follows:

* Only the part of the front-end impacted by the pedestrian needs to be modelled. In practice, a 1 m wide zone was selected to cover the complete pedestrian contact area during the impact.

* This $1 \mathrm{~m}$ wide zone was modelled using two portions of planes: one for the windshield and one for the fairing. These were positioned using the geometric parameters listed in Figure 1 (left).

For the simulations, the two portions of planes were divided into parts of approximately $0.4 \mathrm{~m}$ high. Each part was rigid and its displacement was constrained along its normal direction by a slide connection. Each part was given a mass and attached to a spring to define an equivalent compressive stiffness (Figure 1(right)). This division approach was selected to prevent engaging the whole windshield or the whole fairing at once (as a single rigid body with a large mass and stiffness), and to allow regional compression at the level of the anatomical regions which are engaged. 
A facetted model was used for each part of the frontend, and a finite element to multibody contact was used between each part and the pedestrian model. For rigid body contact handling, each part was meshed with a rectangular grid. A 25 $\mathrm{mm}$ distance between points was used to avoid small ellipsoids of the pedestrian (e.g. wrist) getting locked between nodes of the mesh. Thickness, density and stiffness for the windshield and fairing were defined using technical data shared by the working group as a guide:

* windshield: the thickness was set to $9.3 \mathrm{~mm}$ with a density of $2500 \mathrm{~kg} / \mathrm{m}^{3}(9.3 \mathrm{~kg}$ for each part of $0.4 \mathrm{~m}$ by $1 \mathrm{~m})$ and the spring stiffness was set to $2000 \mathrm{~N} / \mathrm{mm}$ for each part.

* fairing: the thickness was set to $5 \mathrm{~mm}$ with a density of $1000 \mathrm{~kg} / \mathrm{m}^{3}$ ( $2 \mathrm{~kg}$ for each part of $0.4 \mathrm{~m}$ by $\left.1 \mathrm{~m}\right)$ and the spring stiffness was set to $130 \mathrm{~N} / \mathrm{mm}$ for each part.

The same loading and unloading linear elastic characteristics were assumed for all springs. A $20 \mu$ s time step was used for all simulations.

The pedestrian was struck on its left side (struck leg behind) by the tramway moving at a constant speed of $20 \mathrm{~km} / \mathrm{h}$ $(5.45 \mathrm{~m} / \mathrm{s})$. This velocity was selected by the STRMTG to represent a typical pedestrian accident. The tramway braking was not considered. The lateral impact of the pedestrian is consistent with accident data published by Weber et al (2015) in which 18 out of 25 severely and fatally injured persons were hit close to the center of the tramway front when crossing the track. The pedestrian stance was selected to represent a walking pedestrian but the walking speed was not considered.

\section{Parametrization of the Front-end and Simulation Plan}

The range of each geometrical parameter was defined with the support of the working group in an attempt to represent many tramway front-ends in use and to include hypothetical designs as well.

Using two to four values per parameter (deviation angle $\alpha\left(0^{\circ} ;-15^{\circ} ;-30^{\circ} ;-45^{\circ}\right)$; fairing angle $\beta_{\mathrm{F}}\left(0^{\circ} ; 15^{\circ} ; 30^{\circ} ; 45^{\circ}\right)$; windshield angle $\beta_{\mathrm{W}}\left(-10^{\circ}, 0^{\circ} ; 15^{\circ} ; 30^{\circ} ; 45^{\circ}\right)$; windshield offset $\mathrm{d}_{\mathrm{W}} \quad(0 \mathrm{~m} ; 0.1 \mathrm{~m} ; 0.2 \mathrm{~m})$; windshield height $\mathrm{h}_{\mathrm{W}}$ $(0.8 \mathrm{~m} ; 1.05 \mathrm{~m} ; 1.3 \mathrm{~m})$; fairing height $\left.\mathrm{h}_{\mathrm{F}}(0.2 \mathrm{~m} ; 0.35 \mathrm{~m})\right)$ and a full factorial design, 1440 tramway front-end geometries were generated. This full factorial design was applied to the four pedestrian models.

Additional simulations conducted only with the M50 included the application of the full factorial design at $30 \mathrm{~km} / \mathrm{h}$, and intermediate levels for the lower windshield height $\mathrm{h}_{\mathrm{W}}(1.10 \mathrm{~m}, 1.15 \mathrm{~m}, 1.20 \mathrm{~m}$ and $1.25 \mathrm{~m})$ at $20 \mathrm{~km} / \mathrm{h}$. The $30 \mathrm{~km} / \mathrm{h}$ velocity was selected to investigate if the trends observed at $20 \mathrm{~km} / \mathrm{h}$ could still be valid at a higher velocity.

Custom Python scripts were used to automatically generate the MADYMO input files, launch the simulation and postprocess the results. Outputs included the injury criteria available for the MADYMO pedestrian. The HIC15 was used for the head, which is the most common serious and fatal injured in pedestrian to tramway impacts (Weber et al., 2015). Based on (Mertz et al., 1996), an injury assessment reference value of 1000 was used for the HIC: this corresponds to a 15 percent risk to sustain an AIS4+ head injury. For the clustering based analysis, a percentage of simulations with a HIC value below 1000 was computed. It will be referred to as the $\% \mathrm{HIC}<1000$. For other regions, the $\mathrm{V}^{*} \mathrm{C}$ and thorax compression, lateral femur forces and bending moment, lateral knee bending angle and shearing displacement, lateral tibia accelerations were used for the thorax, femur, knee, tibia, respectively. Thresholds were selected based on (EEVC WG17, 2002) and (Schmitt et al., 2004). More details for the selected injury criteria with corresponding thresholds are given in Appendix 2. 
A total of 8,840 simulations were run. Computation time was approximately one minute per run (Processor Intel Core i7-4790 CPU@3.6GHz). The head of the M50 hit the windshield of the tramway in most of the configurations (80\% at $20 \mathrm{~km} / \mathrm{h} ; 90 \%$ at $30 \mathrm{~km} / \mathrm{h}$ ). When analyzing the pedestrian kinematics in detail, two antagonist mechanisms affecting impact velocity of the head were observed. The first was a trunk rotation resulting from an engagement of the lower body, (e.g. for fairing height lower than $1.10 \mathrm{~m}$, Figure 2 left) that can contribute to an increase of the head velocity in the direction of the tram. In that case, the head impact velocity relatively to the tramway front-end can be higher than the tramway velocity, resulting in high HIC values as the HIC was closely related to the head impact velocity (Figure 3). The second mechanism was the loading of the shoulder that can accelerate the upper trunk and head away from the windshield (Figure 2 center), resulting in lower impact velocities and HIC values. In a few cases with shoulder engagement and a large distance between fairing and windshield, the head did not impact the windshield (Figure 2 right). Associated HIC values were low.

The effects of the six parameters describing tramway geometry are shown in Appendix 3 for the M50 impacted at 20 $\mathrm{km} / \mathrm{h}$. No clear tendencies could be observed on isolated parameter plots due to the dispersion of the data. However, when looking at crossed effects between windshield height $h_{w}$ and windshield offset $d_{w}$ (Figure 4), tendencies in line with the previous kinematic analysis on head velocity can be observed: HIC values increased with the windshield offset (up to $0.2 \mathrm{~m}$ ) for windshield heights of $0.8 \mathrm{~m}$ and $1.05 \mathrm{~m}$ but the tendency was reversed for a windshield height of $1.3 \mathrm{~m}$.

Figure 5 represents the HIC cumulative diagram resulting from the full factorial design for each pedestrian size at 20 $\mathrm{km} / \mathrm{h}$ and also at $30 \mathrm{~km} / \mathrm{h}$ for the M50. The HIC median value is below the threshold of 1000 for all pedestrian sizes. The HIC is higher than 1000 for about 30\% of the simulated configurations with the F05 and M50 and for about 15\% with the M95 and C06. The HIC dispersion is smaller for these two last pedestrian sizes. As reported in appendix 4, the thoracic injury criteria were largely below the thresholds for all simulations (e.g. compression below $5 \%$ ). For the lower extremities, the criteria were closer to the thresholds and while were generally below, they were exceptions for all pedestrian sizes. Values exceeded the threshold mainly for the femur moment for the M95, the knee shear for the F05 and M95, and the tibia acceleration mostly for the M50. At $30 \mathrm{~km} / \mathrm{h}$ for the M50, the HIC was higher than 1000 for $75 \%$ of the tramway geometries with a median value around 3000 . Other injury criteria were less affected by the impact velocity, except for the tibia acceleration and in a few cases for the femur force (see appendix 4)

As the windshield height $h_{W}$ and windshield offset $d_{W}$ were the parameters affecting the most the kinematics and loading mechanisms, the two parameters were used to define the groups of design. One hundred and sixty simulations were run for each combination of $h_{w}$ and $d_{w}$, speed and pedestrian size. The percentages of simulations with a HIC below 1000 were computed for each combination and summarized in Table 1. The percentages varied between $100 \%$ (all corresponding designs led to $\% \mathrm{HIC}<1000$ ) and $0 \%$ (for the M50 at $30 \mathrm{~km} / \mathrm{h}$, meaning all designs led to $\%$ HIC >1000). Results with $h_{w}$ of 1.3 were overall better than at other levels, but cross effects between $h_{w}$ and $d_{w}$ were also clearly visible.

\section{DISCUSSION}

A simplified methodology was developed to study the effect of tramway front-end shape on pedestrian injury metrics during simulated accidents. The approach was automated, allowing the systematic exploration of tramway shapes in conjunction with pedestrian size.

Results suggested that the pedestrian head was the most commonly injured body region while the thorax seemed the least exposed. Lower extremities injuries were in between, with patterns that differed between model sizes (e.g. more tibia acceleration for the M50 vs more knee shear for F05 and M50). This is believed to be related to the relative 
positions between knee and fairing height. A high variability was observed for the output variables and, individually, the geometrical parameters selected to describe the front-end were not closely related to the performance in terms of injury criteria. However, combinations of height and offset of the windshield were observed to have an effect on the kinematics and the HIC values. This can be explained by two antagonist mechanisms acting on the head impact velocity relatively to the tramway: the trunk rotation (increasing the velocity) and the shoulder loading (reducing it).

These mechanisms were affected by the pedestrian size. At $20 \mathrm{~km} / \mathrm{h}$, while most of the configurations led to HIC values below 1000 for all the pedestrians, important differences were found depending on the pedestrian size and the shoulder engagement. For example, in the group $\left(\mathrm{h}_{\mathrm{w}}, \mathrm{d}_{\mathrm{w}}\right)=(0.8 \mathrm{~m}, 0 \mathrm{~m})$ corresponding to a windshield height of $0.8 \mathrm{~m}$ and no windshield offset, the HIC is smaller than 1000 in 100\% of the cases for the M95 pedestrian but only $87 \%$ for the M50, $73 \%$ for the F05 and $66 \%$ for the C06. Conversely, configurations in $\left(h_{w}, d_{w}\right)=(1.3 \mathrm{~m}, 0.2 \mathrm{~m})$ had HIC values under 1000 in most cases (100\% for the M50, F05 and C06 and 79\% for the M95).This group seems to be the most robust choice (to both front-end parameters and pedestrian size). It is also consistent with the results of (Weber et al. 2015) suggesting a fairing height of $1.3 \mathrm{~m}$ with offset about $0.08-0.1 \mathrm{~m}$. This study underlines the need to include several pedestrian sizes to choose a strategy or to eliminate configurations which are too dangerous for at least one pedestrian size: for example the configuration $\left(\mathrm{h}_{\mathrm{w}}, \mathrm{d}_{\mathrm{w}}\right)=(0.8 \mathrm{~m}, 0.2 \mathrm{~m})$ had a majority of $\mathrm{HIC}<1000$ for all pedestrian sizes except the F05 for which it is only $14 \%$. Also, in some cases, the kinematics was found to be very sensitive to the front-end geometry even for a single pedestrian size (perhaps due to the shoulder engagement). This can lead to large variations of $\% \mathrm{HIC}<1000$ for limited geometrical changes (e.g. Table 1 for the M50 at $20 \mathrm{~km} / \mathrm{h}$ : the $\% \mathrm{HIC}<1000$ at $\mathrm{d}_{\mathrm{w}}=0.2 \mathrm{~m}$ varies from $100 \%$ down to $15 \%$ and back to $53 \%$ with changes of $h_{w}$ ). Consequently interpolating results between the groups seems questionable and optimizing a design strategy with a single pedestrian size too. This also suggests that while the study can be useful to exclude some groups of geometrical designs or to point design parameters that seems especially favorable (e.g. $h_{w}=1.3 m, d_{w}=0.2 m$ ), it does not ensure the performance of any specific design, especially if not well described by the parameters used in the current study. In general, $\% \mathrm{HIC}<1000$ not equal to $100 \%$ should be considered with care as it can also include very unfavorable designs. The verification of specific designs by simulation using a larger family of pedestrian sizes could also be desirable.

For the M50, the effect of a tramway velocity increased to $30 \mathrm{~km} / \mathrm{h}$ seemed more important on the head injury risk than the effect of pedestrian size at $20 \mathrm{~km} / \mathrm{h}$. It also affected all geometrical combinations (Table 1). At $30 \mathrm{~km} / \mathrm{h}$, the best configuration was for $\left(\mathrm{h}_{\mathrm{w}}, \mathrm{d}_{\mathrm{w}}\right)=(1.3 \mathrm{~m}, 0.2 \mathrm{~m})$ but it only had a $\% \mathrm{HIC}<1000=73 \%$. Within that configuration, a $\% \mathrm{HIC}<1000$ of $100 \%$ was reached only if the windshield angle $\beta_{\mathrm{w}}$ is greater or equal to $15^{\circ}$. These results were attributed to the high stiffness of the tramway windshield as a higher velocity $(40 \mathrm{~km} / \mathrm{h})$ is used for automobiles with the same HIC threshold. This suggests that windshield stiffness reductions may be needed to further improve the results but these may require regulatory changes. Also, only injury criteria that were widely used in the past in the context of tramway safety were selected for the current study. For the head, this led to the use of the HIC. The HIC has been suggested as a good predictor for skull fractures and is used in current regulations for occupant and pedestrian safety. However, limitations have also been pointed for other types of head injuries and alternative injury criteria based on angular motion have been proposed (Takhounts et al., 2013). Such criteria should also be considered in future investigations about tramway safety.

The results of this study have to be considered with some limitations, some of which are related to the initial assumptions. The shoulder engagement was found to be an important factor to decrease the head impact velocity. However, all simulations were run with the pedestrian at 90 degrees with respect to the trajectory of the tramway to 
represent a typical case of pedestrian crossing. The shoulder engagement (and the kinematics) may be affected by this angle and more generally the posture of the pedestrian. The analysis of the results in terms of deviation angle $\alpha$ could provide some insight on this effect but more generally, it is not clear if the shoulder modelling in the MADYMO pedestrian captures well the effect of impact angle on the shoulder response (e.g. as described in (Compigne et al. 2004)). As a perspective, it could be interesting to apply the same approach with finite elements human body models to check if similar tendencies can be observed.

One important limitation of the current study is the relative lack of validation of the tramway model. While wide ranges of geometrical parameters were selected to try to cover current and future designs, the response of the parametrized model was not evaluated against a population of existing designs. Reasons for this lack of validation include the absence of available experimental data and further work should be considered in the future.

Another limitation is that the tramway front-end geometry is represented by two flat surfaces with the same deviation angle $\alpha$, while many tramways present now curved surfaces in both vertical and lateral directions. The angle $\alpha$ aims to represent the orientation of the tramway surfaces at the pedestrian impact location, and different $\alpha$ values can be understood as different frontend designs or different impact locations on the same tramway. However, while constant values of $\alpha$ were used, $\alpha$ would vary along $\mathrm{Z}$ in designs for which the curvature vary along $\mathrm{Z}$. For the curvature in the plane perpendicular to Y, (Weber et al. 2015) suggested that for children, a round shape in the lower part could contribute to accelerate the body towards the ground, increasing the injury risk at the secondary impact. It may also affect the impact location through the wrap around distance. The effect of these simplifications could be studied using a more detailed tramway model, for example introducing an angle $\alpha$ that would vary with $Z$, perhaps reducing the range of other parameters based on the current results. The effect of the secondary impact and possible over-run could also be considered based on a more limited set of parameters.

Finally, the simplification of the tramway by region was implemented to prevent engaging the whole face at one as its mass would be predominant over its stiffness. However, it may not represent well the effect of mass recruitment and the increased stiffness near mounting points (e.g. the edges of the windshield). The use of a finite element model for the front-end (perhaps with a finite element human body model) would help study these mechanisms.

As a conclusion, a wide range of possible tramway shapes was explored in 8,840 simulations with four sizes of pedestrian models. It was found that the injury risk is more important for the head than for the other body regions (thorax and lower extremities).

It was observed that the tramway shape affected the kinematics prior to head impact, which had a large effect on the injury risk. The risk was also very dependent on the pedestrian size, and even more on the tramway velocity.

Overall, the best strategy to limit the risk of head injury due to the contact with the stiff windshield seems to be a combination of shoulder loading by the fairing and windshield offset to limit the head impact velocity. This mechanism seem able to explain many of the differences between groups of designs. At $20 \mathrm{~km} / \mathrm{h}$, a wide majority of configurations leads to HIC $<1000$. As body regions engaged vary with the pedestrian size, none of the groups of designs performed equally well for all pedestrian sizes (e.g. 100\% HIC $<1000$ ). The best compromise seems to be achieved with a combination of a large windscreen offset $(\mathrm{dw}=0.2 \mathrm{~m})$ and a high windscreen $(\mathrm{hw}=1.3 \mathrm{~m})$. Conversely, particularly unfavorable configurations are observed for low windshield heights (below $1.05 \mathrm{~m}$ ), especially with a large offset $(0.2 \mathrm{~m})$. Such designs are not allowed anymore in the frontend design technical guide for new tramways in France (Guesset et al., 2016). 
Beyond the frontend shape, considering the stiffness of the current windshields and the high injury risks predicted at $30 \mathrm{~km} / \mathrm{h}$, reduction of the windshield stiffness and impact speed should be considered in the future for further gains in pedestrian safety.

\section{ACKNOWLEDGEMENT}

This study was funded by the STRMTG. It was conducted in conjunction with a working group comprised of the STRMTG and tramway manufacturer and operators (The list of the working group members can be found in appendix 1 of this document). While the selection of some of the parameters used in the study was performed with the guidance of the working group, the views and results in this study only represent the opinion of the authors and not the opinion of the STRMTG or the working group members. The authors would like to thank Florian Meyer for his contribution to this work during his engineer internship. 


\section{REFERENCES}

Beillas P, Mero M., Belon S., Maupas A., Desfontaines H., Deloffre P., Lapauw P.-Y., Huet L., Charnaux S. Accidents between pedestrians and industrial vehicles: from injury patterns to dummy and truck prototypes. In: 22nd Enhanced Safety of Vehicles Conference (ESV). Washington, USA; 2011.

Compigne S, Caire Y, Quesnel T, Verriest J-P. Non-Injurious and Injurious Impact Response of the Human Shoulder ThreeDimensional Analysis of Kinematics and Determination of Injury Threshold. STAPP Car Crash J. 2004;48:89-125.

Demant AW, Bangard C, Bovenschulte H. MDCT evaluation of injuries after tram accidents in pedestrians. Emerg. Radiol. 2010;17(2):103-108.

EEVC WG17. Improved test methods to evaluate pedestrian protection afforded by passenger cars. European Enhanced VehicleSafety Committee; 2002.

EuroNCAP. Pedestrian Testing Protocole, Version 8.3. 2016.

Feist F, Gugler J., Arregui-Dalmases C., del Pozo de Dios E., Deck C., Willinger R. Pedestrian collisions with flat-fronted vehicles: Injury patterns ans importance of rotational accelerations as a predictor for traumatic brain injury (TBI). In: 21st International Technical Conference on the Enhanced Safety of Vehicles (ESV). Stuttgart, Germany; 2009.

Fredriksson R. Priorities and Potential Pedestrian Protection. Karolinska Institutet, Stockholm; 2011.

Grzebieta RH, Rechnitzer G. Tram Interface Crashworthiness. In: Proc. International Crashworthiness Conference - ICRASH 2000. London, UK; 2000.

Guesset A. Tramway front end design: technical guide. STRMTG; 2016.

Hedelin, A, Björnstig U, Brismar B. Trams—a risk factor for pedestrians. Accid. Anal. Prev. 1996;28(6):733-738.

Hyncik L, Kockova, H., Cihalova, L., Cimrman R. Optimization of tram face with respect to passive safety. Appl. Comput. Mech. 2008:53-62.

Kerrigan J, Arregui C., Crandall J. Pedestrian head impact dynamics: Comparison of dummy and PMHS in small sedan and large SUV impacts. In: 21st International Conference on the Enhanced Safety of Vehicles (ESV). Stuttgart, Germany; 2009.

Kerrigan J, Crandall J, Deng B. Pedestrian kinematic response to mid-sized vehicle impact. Int. J. Veh. Saf. $2007: 221$ - 240.

de Labonnefon V, Passelaigue JM. Rapport annuel sur le parc, le trafic et les évènements d'exploitation des tramways: année 2015, évolution 2006-2015. STRMTG; 2016.

Martinez L, Guerra LJ, Ferichola G, Garcia A, Yang J. Stiffness Corridors of the European Fleet for Pedestrian Simulations. In: 20th International Technical Conference on the Enhanced Safety of Vehicles (ESV). Lyon, France; 2007.

Matsui Y, Oikawa S, Sorimachi K, Imanishi A, Fujimura T. Association of Impact Velocity with Serious-injury and Fatality Risks to Cyclists in Commercial Truck-Cyclist Accidents. STAPP Car Crash J. 2017;61:355-371.

Mertz HJ, Prasad P, Nusholtz G. Head Injury Risk Assessment for Forehead Impacts. SAE Tech. Pap. 960099. 1996.

NF-F15818. Norme NF F15-818 Matériel roulant ferroviaire - Vitres frontales (Railway rolling stock - Frontal windscreens). AFNOR. 1996.

Regulation (EC) No 78. Regulation (EC) No 78/2009 on the type-approval of motor vehicles with regard to the protection of pedestrians and other vulnerable road users, Official Journal of the European Union, L 35. European Parliament, Council of the European Union.; 2009.

Schmitt K.-U., Niederer P. F., Walz F. Trauma Biomechanics - Introduction to Accidental lnjury. 4th Edition. Springer ISBN 9783-662-05448-2 (eBook); 2004. 
Soni A, Robert T, Beillas P. Effects of Pedestrian Pre-Crash Reactions on Crash Outcomes during Multi-body Simulations. In: International Research Council On the Biomechanics of Impact Conference (IRCOBI). Gothenburg, Sweden; 2013.

Subit D, Kerrigan J., Crandall J., Fukuyama K., Yamazaki K., Kamiji K., Yasuki T. Pedestrian-vehicle interaction: Kinematics and injury analysis of four full-scale tests. In: International Research Council On the Biomechanics of Impact Conference (IRCOBI). Bern, Switzerland; 2008.

Takhounts E.G., Craig M.J., Moorhouse K., McFadden J. Development of Brain Injury Criteria (BrIC). In: STAPP Car Crash J. 2013 57:243-266.

TASS. International, MADYMO Reference Model, Release 7.5. 2013.

TNO. Road-Vehicle Research Institute. MADYMO Human Body Models Manual, Release 7.5. 2013.

Van Hoof J, De Lange R, Wisman J. Improving Pedestrian Safety Using Numerical Human Models. SAE Rep. N ${ }^{\circ}$ 2003-22-0018 Stapp Car Crash J. 2003;47:401-436.

Van Rooij L, Bhalla K, Meissner M, Ivarsson J, Crandall J, Longhitano D, Takahashi Y, Dokko Y, Kikuchi Y. Pedestrian crash reconstruction using multi-body modeling with geometrically detailed, validated vehicle models and advanced pedestrian injury criteria. In: 18th International Technical Conference on the Enhanced Safety of Vehicles (ESV). Nagoya, Japan; 2003.

Watanabe R, Katsuhara T., Miyazaki H., Kitagawa Y., Yasuki T. Research of the relationship of pedestrian injury to collision speed, car-type, impact location and pedestrian sizes using human FE model (THUMS Version 4). Stapp Car Crash J. 2012;56:269-321.

Weber T, Muser M., Schmitt K.-U. Optimising the design of tramways to mitigate injury risk in pedestrian impacts. In: International Research Council On the Biomechanics of Impact Conference (IRCOBI). Lyon, France; 2015. 


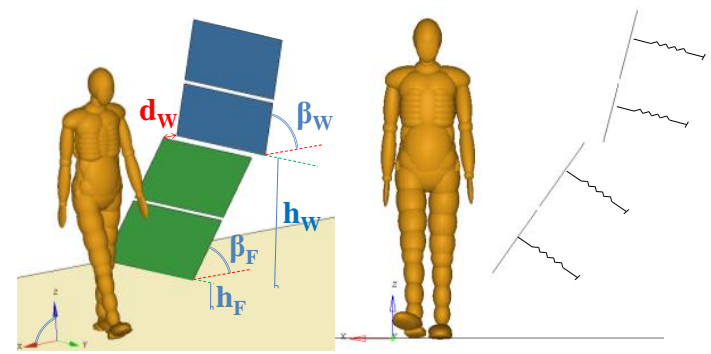

Figure 1 : Tramway front-end modelled using two portions of planes (shown here with a mid-size male model M50 and without rotation of the front-end around the z-axis). The fairing (green) and windshield (blue) are defined by 6 parameters: the fairing and the windshield angles (inclination $\beta_{\mathrm{F}}$ and $\beta_{\mathrm{W}}$ with respect to the vertical, respectively), the height of the lower edge of the fairing (fairing height $h_{F}$ with respect to the ground), the height of the lower edge of the windshield (windshield height $h_{\mathrm{W}}$, with respect to the ground), the horizontal offset between the lower windshield and the upper fairing (horizontal windshield offset $\mathrm{d}_{\mathrm{W}}$ ) and the deviation angle of the front-end (rotation $\alpha$ around the z-axis). The two portions of planes are divided into parts of $1 \mathrm{~m}$ wide and approximately $0.4 \mathrm{~m}$ high meshed with $25 \mathrm{~mm}$ spaced nodes to support contacts and attached to springs with displacement constrained along their normal directions (see the "side view" on the right). A friction coefficient of 0.35 was used between the body and the tram.

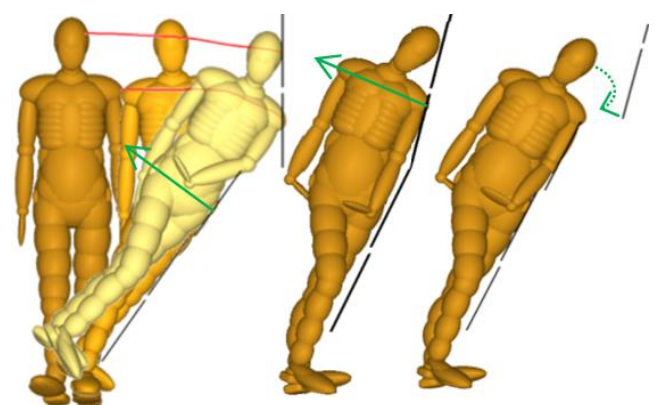

Figure 2: Kinematic mechanisms observed. Left: the trunk rotation, initiated by the engagement of the lower body, can contribute to an increased velocity of the head when it finally reaches the windshield (here: fairing height of $1.05 \mathrm{~m}$, relative head impact velocity of $6.77 \mathrm{~m} / \mathrm{s}$ and HIC of 5520). Center: shoulder loading can contribute to a reduced velocity of the head when it hits the windshield (here: fairing height of $1.05 \mathrm{~m}$, relative head velocity of $1.6 \mathrm{~m} / \mathrm{s}$ and a HIC of 80 ). Right: a shoulder loading by the fairing combined with a windshield offset at the level of the head, can lead to no head impact against the windshield (here fairing height of $1.30 \mathrm{~m}$ and a HIC of 120) - All cases with a tramway velocity of $20 \mathrm{~km} / \mathrm{h}$ and the M50.

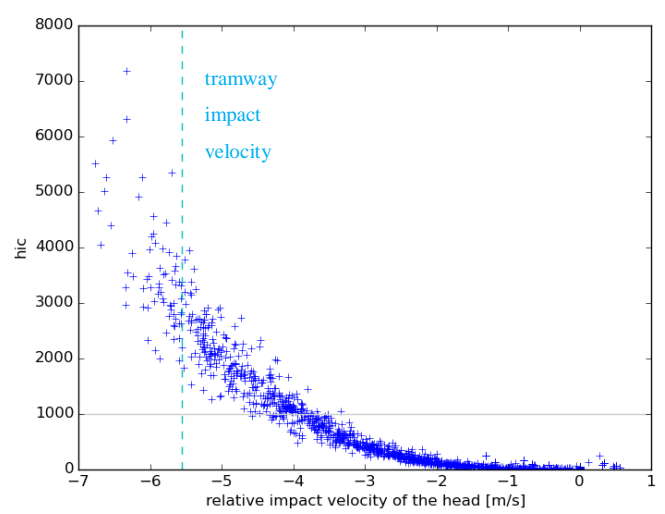

Figure 3 : HIC vs relative impact velocity of the M50 head at $20 \mathrm{~km} / \mathrm{h}(5.45 \mathrm{~m} / \mathrm{s})$. 

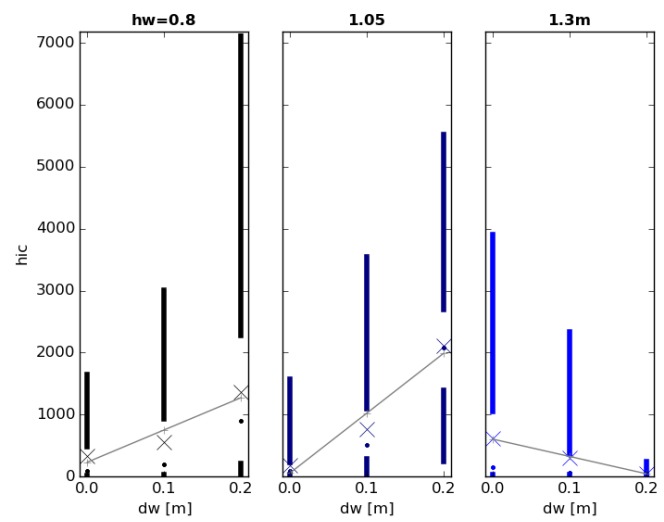

Figure 4 : Cross effect of windshield height $h_{W}$ and windshield offset $d_{W}$ on HIC distribution by quartiles for the M50 impacted by the tramway at $20 \mathrm{~km} / \mathrm{h}$ (box and whiskers plots: the cross is the mean, the dot is the median, thick lines are quartiles Q1 and Q4, the intervals between the thick lines and the median depict quartiles Q2 and Q3). HIC values increase with the windshield offset (up to $0.2 \mathrm{~m}$ ) for windshield heights of $0.8 \mathrm{~m}$ and $1.05 \mathrm{~m}$ but the tendency is reversed for a windshield height of $1.3 \mathrm{~m}$ (pvalue $<0.05)$.
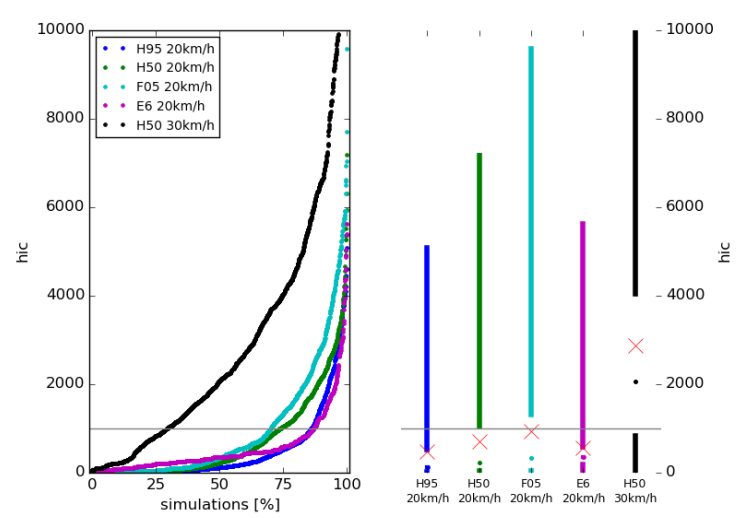

Figure 5 : HIC Cumulative diagrams at $20 \mathrm{~km} / \mathrm{h}$ for each pedestrian size and at $30 \mathrm{~km} / \mathrm{h}$ for the M50 (left) and (right) corresponding distribution by quartiles (box and whiskers plots: the cross is the mean, the dot is the median, thick lines are quartiles Q1 and Q4, the intervals between the thick lines and the median depict quartiles Q2 and Q3) 
Table 1 : Percentage of configurations leading to a HIC below 1000 as function of pedestrian size, tramway speed, windshield height $h_{\mathrm{W}}$ and windshield offset $\mathrm{d}_{\mathrm{W}}$. Each percentage was calculated out of 160 simulations. Ideal designs would have percentages of $100 \%$ for all sizes of pedestrians and velocities. A number below $100 \%$ means that at least one combination of other geometrical parameters led to a HIC higher than 1000 .

\begin{tabular}{|c|c|c|c|c|c|}
\hline \multirow{3}{*}{$\begin{array}{l}\text { Speed } \\
(k m / h)\end{array}$} & \multirow{3}{*}{$\begin{array}{c}\text { Ped. } \\
\text { model }\end{array}$} & \multicolumn{4}{|c|}{ Windshield } \\
\hline & & \multirow{2}{*}{$\begin{array}{l}\text { height } \\
h_{w}(m)\end{array}$} & \multicolumn{3}{|c|}{ offset $d_{w}(m)$} \\
\hline & & & 0 & 0.1 & 0.2 \\
\hline \multirow[t]{16}{*}{20} & M50 & 1.30 & $74 \%$ & $89 \%$ & $100 \%$ \\
\hline & & 1.25 & & $80 \%$ & $99 \%$ \\
\hline & & 1.20 & & $76 \%$ & $88 \%$ \\
\hline & & 1.15 & & $73 \%$ & $74 \%$ \\
\hline & & 1.10 & & $74 \%$ & $28 \%$ \\
\hline & & 1.05 & $98 \%$ & $71 \%$ & $15 \%$ \\
\hline & & 0.80 & $87 \%$ & $77 \%$ & $53 \%$ \\
\hline & $\mathrm{C06}$ & 1.30 & & $100 \%$ & $100 \%$ \\
\hline & & 1.05 & $84 \%$ & $100 \%$ & $100 \%$ \\
\hline & & 0.80 & $66 \%$ & $54 \%$ & $89 \%$ \\
\hline & F05 & 1.30 & $73 \%$ & $100 \%$ & $100 \%$ \\
\hline & & 1.05 & $75 \%$ & $61 \%$ & $85 \%$ \\
\hline & & 0.80 & $73 \%$ & $52 \%$ & $14 \%$ \\
\hline & M95 & 1.30 & $93 \%$ & $80 \%$ & $79 \%$ \\
\hline & & 1.05 & $100 \%$ & $88 \%$ & $41 \%$ \\
\hline & & 0.80 & $100 \%$ & $99 \%$ & $96 \%$ \\
\hline \multirow[t]{3}{*}{30} & M50 & 1.30 & $42 \%$ & $43 \%$ & $73 \%$ \\
\hline & & 1.05 & $34 \%$ & $13 \%$ & $0 \%$ \\
\hline & & 0.80 & $22 \%$ & $21 \%$ & $18 \%$ \\
\hline
\end{tabular}

